\title{
Fasting blood glucose in ACS patients
}

Several studies have shown a correlation between 'admission hyperglycemia'-nonfasting increases in blood glucose levels in hospitalized patients-and increased mortality rates. The GRACE investigators have now reported that elevated fasting blood glucose levels increase the risk of complications for patients with ACS.

The AHA issued a statement in 2008 that highlighted the need to better understand the link between hyperglycemia and adverse outcomes. "This statement was really timely," Peter Sinnaeve, one of the investigators, remarked, "pointing at hyperglycemia in ACS as a known but underestimated risk factor. They identified some important but unanswered questions, including what is the best way to assess hyperglycemia, is hyperglycemia a contributing factor to adverse outcomes or merely a marker of a high risk state, and should we treat hyperglycemia in ACS?"

In designing their study, Sinnaeve et al. noted a distinction between admission hyperglycemia, which reflects a stress response, and the fasting blood glucose level, which is a more accurate indicator of glucose metabolism. Few studies have assessed the effects of fasting blood glucose levels in ACS and it is not clear whether the poorer prognosis associated with hyperglycemia is attributable to a stress response or to dysfunctional glucose metabolism. Both admission and fasting blood glucose levels of up to 22,000 patients were thus analyzed. A strong correlation was found between fasting blood glucose levels and mortality rates-a modest elevation above normal was sufficient to increase risks for patients in hospital and up to 6 months after discharge. By contrast, a correlation between admission hyperglycemia and adverse outcomes was seen only at glucose levels above the threshold for diabetes and only while patients were hospitalized.

The findings of this study suggest that fasting blood glucose levels might be a better indicator of risks for patients with ACS than admission hyperglycemia, irrespective of a history of diabetes. Two key questions now remain: will tight control of blood glucose levels during hospitalization improve the prognosis for patients, and should glucose-lowering measures be introduced (or intensified) after discharge, particularly for patients with 'prediabetic' blood glucose levels?

\section{Sharmini Rajanayagam}

Original article Sinnaeve, P. R. et al. Association of elevated fasting glucose with increased short-term and 6-month mortality in ST-segment elevation and non-ST-segment elevation acute coronary syndromes. Arch. Int. Med. 169, 402-409 (2009). 